\title{
Restoring high accuracy to laser Doppler vibrometry measurements affected by vibration of beam steering optics
}

Ben J. Halkon, Steve J. Rothberg

Wolfson School. Mechanical, Electrical and Manufacturing Engineering

Loughborough University, Loughborough, Leicestershire, LE11 3TU, UK.

Corresponding author: Steve J. Rothberg

e-mail: s.j.rothberg@lboro.ac.uk

tel: +44 (0) 1509223440 


\title{
Restoring high accuracy to laser Doppler vibrometry measurements affected by vibration of beam steering optics
}

\author{
Ben J. Halkon, Steve J. Rothberg
}

Wolfson School. Mechanical, Electrical and Manufacturing Engineering

Loughborough University, Loughborough, Leicestershire, LE11 3TU, UK.

\begin{abstract}
Laser Doppler vibrometers are now well-established as an effective non-contact alternative to traditional contacting transducers. Wide-ranging applications include those where beam steering optics are required to reach locations that are difficult to access but no attention has yet been given to measurement sensitivity to the vibration of those optics. In this paper, a thorough mathematical treatment of this sensitivity to steering optic vibration and its correction is set out. A very practical scheme requiring a single correction measurement, from the back-surface of the mirror at the incidence point and aligned with the mirror normal, delivers an error reduction typically in excess of $30 \mathrm{~dB}$. After validation in the laboratory, the scheme is then applied to a genuinely challenging measurement scenario on a single cylinder racing motorcycle. Correction is theoretically perfect for translational mirror vibrations but angular mirror vibrations require an adapted scheme using a triplet of accelerometers arranged around a circular path on the mirror back-surface and this is set out theoretically.
\end{abstract}

KEYWORDS: laser Doppler vibrometry; vibration measurement; correction; beam steering; measurement uncertainty. 


\section{Introduction}

2 The laser Doppler vibrometer (LDV), the basis for which is the detection of the Doppler frequency

3 shift that occurs when light is scattered by a moving surface, is now well-established as an effective non-contact alternative to traditional contacting vibration transducers such as piezoelectric accelerometers [1]. Commercially available LDVs are technically well suited to general application but they offer special benefits in a variety of challenging measurement scenarios including those in which the measurement point of interest is difficult to access. In such cases, steering optics (typically mirrors) are often used to direct the probe laser beam to the point of interest with the required orientation. Practically, this means positioning the steering optic in close proximity to the measurement point which generally necessitates its mounting at a convenient location near to or even within the structure of interest, without opportunity for vibration isolation. Valve motions in reciprocating engines have been the most commonly reported application [2-4] in which this issue is of particular importance. When the laser beam is incident at a point on any surface at which there is a change in the laser beam direction, the LDV measurement will be sensitive to the vibration of that surface. Consequently, any vibration of a steering optic will result in erroneous measurement content due to Doppler shift that occurs both outbound to and inbound from the target. The specific novel contributions made in this paper will enable complete correction of measured vibration by application of a vector-based approach to determine the sensitivity of LDV measurements to steering optic vibration.

Building on the previously reported proof-of-principle [5], the objectives of this paper are to show theoretically and confirm experimentally how the measured velocity for such scenarios will include a component proportional to the vibration velocity at the point of incidence on an optical device whenever a change in beam direction occurs (through reflection or refraction). The required correction to the LDV measurement is set out theoretically and then demonstrated experimentally for a laboratory-based set-up, in which translational vibration of the steering mirror is arranged. While previously only the effect of steering mirror vibration was explored, this paper extends this to, for the first time, a thorough treatment of the real-world scenario where both steering mirror and target vibration are present. Additionally, a previously unreported genuinely real-world measurement on a single cylinder racing motorcycle engine mounting bracket, in which a steering mirror was needed to direct the laser beam to an inaccessible measurement location, is described with the required correction due to the vibration of the steering mirror being applied. Finally, the paper extends the mathematical description of the LDV measurement, for the first time, to include not just translational but also angular vibrations of a steering mirror. The error associated with angular vibrations in a measurement corrected for only translational vibrations is quantified. Ultimately, a scheme for correction of both translational and angular steering mirror vibration is proposed theoretically for future practical implementation. 
2 LDV is a technique that has built its reputation on real world measurements with high accuracy. The

3 practical steps proposed in this paper, like those proposed in a recent study of the effects of vibration

4 of the instrument itself [6], are essential to restoring that accuracy in the kind of challenging

5 applications that are the trademark of LDV and represent a significant and important contribution to

6 the vibration engineering community.

\section{2. Modelling the measured velocity}

8 To enable general determination of the velocity measured for any arrangement, knowledge of the 9 points of incidence on all moving surfaces, at which a change in laser beam direction occurs, is 10 required [7]. For the arrangement under consideration here, this means both the vibrating steering 11 optic and the target itself. The modelling task begins with a vector description of the outgoing laser 12 beam direction and an arbitrary point through which the laser beam passes, as shown in Figure 1, for 13 the laser beam direction:

$$
\hat{b}_{1}=-\hat{x}
$$

[INSERT: Figure 1. Schematic showing translational vibration measurement using an angled steering mirror; beam deviation significantly exaggerated for the sake of clarity]

An arbitrarily chosen point, denoted A, along the line of the laser beam can be written in vector form:

$$
\overrightarrow{O A}=\left[\begin{array}{lll}
\hat{x} & \hat{y} & \hat{z}
\end{array}\right]\left[\begin{array}{lll}
x_{A} & y_{A} & z_{A}
\end{array}\right]^{T}
$$

For an arrangement in which a plane mirror is used to align and position the laser beam onto the point of interest on the target surface, mathematical description of the mirror location, incident point and its normal are required. With reference to Figure 1, an arbitrary reference point on the mirror (in the absence of vibration), denoted $\mathrm{B}$, can be expressed generally as:

$$
\overrightarrow{O B}=\left[\begin{array}{lll}
\hat{x} & \hat{y} & \hat{z}
\end{array}\right]\left[\begin{array}{lll}
x_{B} & y_{B} & z_{B}
\end{array}\right]^{T}
$$

The figure shows the path taken to the target (dotted line) in the absence of mirror vibration. The beam is shown steered through 90 degrees, though this is just an arbitrary choice. The plane of the target (in the absence of vibration) includes the origin, $\mathrm{O}$, of the global coordinate system though this is, again, just an arbitrary choice.

When the mirror undergoes arbitrary six degree-of-freedom vibration, the level of which is significantly exaggerated in Figure 1 for the sake of clarity, the point $B$ moves to $B^{*}$ where:

$$
\overrightarrow{O B^{*}}=\overrightarrow{O B}+\left[\begin{array}{lll}
\hat{x}_{m} & \hat{y}_{m} & \hat{z}_{m}
\end{array}\right]\left[\begin{array}{lll}
B B^{*}{ }_{m x} & B B^{*}{ }_{m y} & B B^{*}{ }_{m z}
\end{array}\right]^{T}
$$


1 in which $\left[\begin{array}{lll}\hat{x}_{m} & \hat{y}_{m} & \hat{z}_{m}\end{array}\right]$ is the coordinate system for the mirror and $\left[\begin{array}{llll}B B^{*}{ }_{m x} & B B^{*}{ }_{m y} & B B^{*}{ }_{m z}\end{array}\right]$ are 2 the components of the vector $\overrightarrow{B B^{*}}$, from point $B$ to $B^{*}$, in the mirror coordinate system. The surface

3 normal for the mirror, $\hat{n}_{B *}$, can be written easily in terms of the mirror coordinate system as:

$$
\hat{n}_{B *}=\left[\begin{array}{lll}
\hat{x}_{m} & \hat{y}_{m} & \hat{z}_{m}
\end{array}\right]\left[\begin{array}{ccc}
0 & 0 & -1
\end{array}\right]^{T}=\hat{z}_{m}
$$

5 With reference to the rotation matrices defined in the Appendix A, the mirror coordinate system can

6 be linked to the global system by the transformation:

$$
\left[\begin{array}{lll}
\hat{x}_{m} & \hat{y}_{m} & \hat{z}_{m}
\end{array}\right]=\left[\begin{array}{lll}
\hat{x} & \hat{y} & \hat{z}
\end{array}\right]\left[x, \theta_{m x}\right]\left[y, \theta_{m y}\right]\left[\begin{array}{lll}
1 & 0 & 0 \\
0 & 1 & 0 \\
0 & 0 & 1
\end{array}\right]
$$

such that the mirror normal can be rewritten in terms of the global coordinate system as:

$$
\hat{n}_{B *}=\left[\begin{array}{lll}
\hat{x} & \hat{y} & \hat{z}
\end{array}\right]\left[x, \theta_{m x}\right]\left[y, \theta_{m y}\right]\left[\begin{array}{lll}
0 & 0 & -1
\end{array}\right]^{T}
$$

The angles $\theta_{m x}$ and $\theta_{m y}$ combine initial alignment, $\alpha_{m}$ and $\beta_{m}$, and subsequent vibrations, $\Delta \theta_{m x}$ and $11 \Delta \theta_{m y}$ which can be written explicitly as:

$$
\hat{n}_{B *}=\left[\begin{array}{lll}
\hat{x} & \hat{y} & \hat{z}
\end{array}\right]\left[x, \alpha_{m}+\Delta \theta_{m x}\right]\left[y, \beta_{m}+\Delta \theta_{m y}\right]\left[\begin{array}{lll}
0 & 0 & -1
\end{array}\right]^{T}
$$

The laser beam direction after reflection at the mirror can then be written as:

$$
\hat{b}_{2}=\hat{b}_{1}-2\left(\hat{b}_{1} \cdot \hat{n}_{B *}\right) \hat{n}_{B *}
$$

Vibration of the mirror affects the location, $B^{\prime}$, at which the laser beam is incident on the mirror surface. This point can be obtained from the following set of equations. The first is a vector triangle involving the laser beam path to the mirror:

$$
\overrightarrow{O B^{\prime}}=\overrightarrow{O A}+\left|\overrightarrow{A B^{\prime}}\right| \hat{b}_{1}
$$

The second is a vector triangle involving the instantaneous locations of the reference position on the mirror during vibration, $B^{*}$, and the point of incidence on the mirror, $B^{\prime}$ :

$$
\overrightarrow{O B^{\prime}}=\overrightarrow{O B^{*}}+\overrightarrow{B^{*} B^{\prime}}
$$

22 The third recognises that the vector $\overrightarrow{B^{*} B^{\prime}}$ lies in the plane of the mirror:

$$
\overrightarrow{B^{*} B^{\prime}} \cdot \hat{n}_{B^{*}}=0
$$


1 These equations can be combined and lead to:

2

$$
\overrightarrow{O B^{\prime}}=\overrightarrow{O A}+\left[\frac{\left(\overrightarrow{O B^{*}}-\overrightarrow{O A}\right) \cdot \hat{n}_{B^{*}}}{\hat{b}_{1} \cdot \hat{n}_{B^{*}}}\right] \hat{b}_{1}
$$

The point $T^{\prime}$ is found in slightly simpler fashion, using just two vector equations. The first is the vector triangle involving the laser beam path while the second recognises that the position $\overrightarrow{O T^{\prime}}$ lies in the $x y$ plane:

$$
\overrightarrow{O T^{\prime}}=\overrightarrow{O B^{\prime}}+\left|\overrightarrow{B^{\prime} T^{\prime}}\right| \hat{b}_{2}
$$

leading to:

$$
\overrightarrow{O T^{\prime}} \cdot \hat{z}=0
$$

$\overrightarrow{O T^{\prime}}=\overrightarrow{O B^{\prime}}-\left[\overrightarrow{\frac{O B^{\prime}}{.} \hat{z}}\right] \hat{b}_{2}$

While the intention is of course to measure the target vibration in isolation, the total measured velocity is clearly the sum of the measured velocities from both points $T^{\prime}$ and $B^{\prime}$ and is written as:

$$
U_{m}=-\hat{b}_{2} \cdot \overrightarrow{V_{T^{\prime}}}+\left(\hat{b}_{2}-\hat{b}_{1}\right) \cdot \overrightarrow{V_{B^{\prime}}}
$$

where $\overrightarrow{V_{B^{\prime}}}$ is a combination of the translational motion of the reference point $B^{*}$ and rotational motion around that point. In fact, the LDV probe beam is Doppler shifted twice at the mirror surface, once on the way to and again on the way back from the target surface. Both shifts are included in equation (10).

The first term in equation (10) is the intended measurement but there are secondary effects on this quantity as a result of mirror vibration. The point $B$ ' changes continuously during mirror vibration and this causes the instantaneous point on the target at which the laser beam is incident, $T^{\prime}$, to move correspondingly. Figure 1 exaggerates the movement of the laser beam on the target in order to emphasise the point that the mirror vibration results in illumination of a small region on the target that in practice is slightly larger than the incident laser beam. Furthermore, small deviations occur in the incident beam direction that affect the components of vibration sensed. Neither of these small effects is compensated for in the procedure set out here. Effectively, two very reasonable assumptions are made. The first is that the vibration of the target does not vary within the small illuminated region, a region that is generally still much smaller than the size of a contacting transducer. The second assumption is that the angular deviation of the laser beam has a negligible effect on measured 
1 velocity, which is reasonable considering that even an improbably large deviation of 5 degrees would

2 only result in an error of less than $0.5 \%$ in the measured, $z$-direction, target velocity.

3 The correction procedure is focussed wholly on the much more significant second term in equation

4 (10) that is directly related to the velocity at $B$ '. Substituting equation (7) and then equation (4) into

5 this term, the measured velocity due to incidence at the point B' on the mirror, $U_{m B^{\prime}}$, can be written

6 as:

7

$$
U_{m B^{\prime}}=-2\left(\hat{b}_{1} \cdot \hat{n}_{B *}\right) \hat{n}_{B *} \cdot \overrightarrow{V_{B^{\prime}}}=-2\left(\hat{b}_{1} \cdot \hat{n}_{B *}\right)\left(\hat{z}_{m} \cdot \overrightarrow{V_{B^{\prime}}}\right)
$$

8 To eliminate the influence of the mirror vibration on the measured LDV signal and yield the intended 9 measurement of target surface vibration only, the goal is clearly to measure $U_{m B^{\prime}}$ such that it can be 10 subtracted from the total LDV measurement made. Equation (11), specifically the dot product $11\left(\hat{z}_{m} \cdot \overrightarrow{V_{B^{\prime}}}\right)$, shows that this can be achieved to a very large extent by a single velocity measurement at 12 the point of laser beam incidence, aligned in the $\hat{z}_{m}$ direction. This is a very significant result because 13 it points towards a simple and practical solution to the problem using a single transducer, attached to 14 the back-surface of the mirror and aligned with the incidence location, to effect the required correction. A contacting transducer is ideal because it maintains its alignment with the mirror normal even during mirror angular vibrations. Such correction will be perfect for translational vibrations and this is explored experimentally in the following section. The paper returns to the issue of angular vibrations in section 5 .

\section{Experimental investigation - laboratory-based measurements}

\section{Experimental arrangement}

The experimental arrangement for laboratory tests is shown in Figure 2. The target and steering mirror translational motions were generated independently by small electrodynamic shakers mounted horizontally with rods connecting the armatures to small assemblies mounted atop low-friction linear bearings. Uncorrelated broadband vibrations, to emphasise the versatility of the method, were excited by each shaker with levels chosen to be representative of what might be encountered in real-world applications. The LDV (Polytec OFV4000 set-up for translational vibration measurement), which can just be seen in the foreground towards the bottom right of the image, was tripod-mounted on the floor adjacent to the vibration isolated optical table on which the various other hardware items were positioned.

[INSERT: Figure 2. Equipment arrangement for laboratory-based experimental investigation; laser beam path highlighted for the sake of clarity] The probe laser beam, nominally in a horizontal plane, was steered through the required angle $\left(90^{\circ}\right.$ in the configuration shown but the arrangement was readily re-configurable for alternative angles) by the 
1 mirror mounted on a vertical face of the assembly. One shaker drives mirror vibration in the direction

2 of the mirror normal, measured by a piezoelectric accelerometer mounted on the reverse side of the

3 mirror and positioned directly behind the point of laser beam incidence. The motion of the target,

4 nominally coincident with the laser beam direction, was similarly determined by attachment of a

5 piezoelectric accelerometer, in this case on the vertical front face of the target assembly; this provides

6 the 'true' measurement. The LDV - target stand-off distance was $400 \mathrm{~mm}$, in line with the

7 manufacturer's recommendation, with the probe laser beam targeted directly onto the top of the

8 accelerometer so as to enable direct comparison between the two measurements.

9 As the first stage of the measurement campaign, both target and mirror accelerometer amplitude

10 sensitivities were cross-checked against the LDV using a broadband (white noise) vibration

11 characteristic and adjusted accordingly. In the case of the mirror accelerometer, this was achieved by

12 temporarily attaching it to the top of the target accelerometer such that both accelerometers and the

13 LDV were exposed to exactly the same target motion.

14 To achieve the necessary subtraction between LDV and accelerometer measurements, inter-channel

15 time delays must be accounted for. As shown in Figure 3, such time delays result in a characteristic

16 phase error that is directly proportional to frequency. For a broadband vibration, the mean time delay

17 (across the frequency range of interest) between the LDV and (integrated) accelerometer channels can

18 be calculated from the gradient of the plot, and the phase error can then be eliminated [5,6], as also

19 shown in Figure 3. Such offline processing was undertaken in the frequency, rather than time, domain

20 for reasons of the relative simplicity and accuracy of integration of the accelerometer outputs for

21 direct comparison with that of the LDV, correction of inter-channel time delays, subsequent

22 subtraction of signals, and averaging.

23 [INSERT: Figure 3. Example phase difference between LDV measurement and integrated

24 accelerometer measurement before and after time delay adjustment]

Validating the measurement correction theory

With reference to the required correction set out theoretically in the previous section, for the arrangement shown in Figure 2, $\alpha_{m}$ is zero and the angular vibrations are also zero in this experimental validation. Hence, equation (6b) can be written as:

$$
\hat{n}_{B *}=-\sin \beta_{m} \hat{x}-\cos \beta_{m} \hat{z}
$$

30 Combination of equations (1), (11) and (12), allows the corrected velocity measurement, $U_{c o r r}$, to be 31 written as:

$$
U_{\text {corr }}=U_{m}-U_{m B^{\prime}}=U_{m}+2 \sin \beta_{m}\left(\hat{z}_{m} \cdot \overrightarrow{V_{B^{\prime}}}\right)
$$


1 Sensitivity to the component of steering mirror vibration in the direction of the mirror normal, as set

2 out in equation (11), is readily observed by a scenario in which there is mirror vibration but nominally

3 zero target vibration. As shown in Figure 4, the actual target vibration is at an extremely low level as

4 expected but the measurement from the LDV, with its beam steered with $\beta_{m}=-\frac{\pi}{4}$, shows significant

5 sensitivity to the vibration of the mirror itself. The measurement is two to three orders of magnitude

6 higher than actual target vibration.

7 [INSERT: Figure 4. Comparison between measurements from the LDV and from the target

8 accelerometer on a (nominally) stationary target (beam steered through $90^{\circ}$ by the mirror with

$9 \quad \beta_{\mathrm{m}}=-\frac{\pi}{4}$ rad; mirror vibration at $50 \times 10^{-3} \mathrm{~g}$ RMS broadband)]

Making use of the time-delay adjusted and integrated steering mirror accelerometer signal to perform the required correction as set out in equation (13), a significant correction, as shown in Figure 5a, is achieved. The dB error reduction, shown in Figure 5b, has a mean level in excess of $35 \mathrm{~dB}$. Set-ups with the beam steered through $60^{\circ}$, i.e. with $\beta_{m}=-\frac{\pi}{3}$, and $30^{\circ}$, i.e. with $\beta_{m}=-\frac{5 \pi}{12}$, were also arranged with equivalent findings obtained in terms of correction performance as demonstrated in Figure 6. The solution proposed is completely general and easy to implement provided the mirror orientation can be determined.

[INSERT: Figure 5. Comparison between measured and corrected LDV measurements with respect to the 'true' target vibration (stationary target, beam steered through $90^{\circ}$ by the mirror with $\beta_{\mathrm{m}}=-\frac{\pi}{4}$ rad; mirror vibration at $50 \times 10^{-3} \mathrm{~g}$ RMS broadband); mean a) amplitudes and b) dB reduction] [INSERT: Figure 6. Comparison between measured and corrected LDV measurements with respect to the 'true' target vibration (stationary target, mirror vibration at $50 \times 10^{-3} \mathrm{~g}$ RMS broadband); a) beam steered through $60^{\circ}$, i.e. $\beta_{\mathrm{m}}=-\frac{\pi}{3} \mathrm{rad}$, b) beam steered through $30^{\circ}$, i.e. $\beta_{\mathrm{m}}=-\frac{5 \pi}{12} \mathrm{rad}$ and c) $\mathrm{dB}$ reduction]

\section{Correction for simultaneous steering mirror and target vibration}

A second laboratory experiment was conducted using the same experimental arrangement as shown in Figure 2, although this time with both target ( $30 \times 10^{-3} \mathrm{~g}$ RMS broadband) and steering mirror vibration (50 x $10^{-3} \mathrm{~g}$ RMS broadband) applied. This scenario is representative of the kind of situation which might be experienced in a "real world" application, for example when a mirror is mounted to a vibrating surface to enable the probe laser beam to be steered toward a vibrating target surface of interest but for which access is limited. As expected, substantial differences are observed, as shown in Figure 7a, between the measurement from the LDV (dashed curve) and the 'true' measurement from the target accelerometer (dotted curve). After correction, however, excellent agreement is found, also shown in Figure 7a, between the corrected measurement (solid curve) and the 'true' vibration. In fact, 
1 the corrected and true measurements are almost indistinguishable in Figure 7a so their comparison is

2 shown in Figure $7 \mathrm{~b}$ in the form of a dB error reduction; a mean of over $47 \mathrm{~dB}$ is found. Excellent

3 agreement is also found between the phase of the corrected measurement and the 'true' measurement,

4 as shown in Figure 7c (RMS error $26 \mathrm{mrad}$ ). Similar results were found for other beam steering angles

5 and different ratios of target: mirror vibration. For example, Figure 8 shows data for $\beta_{m}=-\frac{5 \pi}{12}$

6 (beam steered through $30^{\circ}$ ), steering mirror vibration at $50 \times 10^{-3} \mathrm{~g}$ RMS broadband and target

7 vibration at $60 \times 10^{-3} \mathrm{~g}$ RMS broadband. At least $33 \mathrm{~dB}$ mean error reduction is observed together

8 with excellent phase agreement (RMS error $31 \mathrm{mrad}$ ). These results validate the correction method

9 captured mathematically in equation (11) and implemented practically as described so far in this

10 paper.

11 [INSERT: Figure 7. Comparison between LDV measurement before and after correction with 'true' 12 target vibration (steering mirror vibration at $50 \times 10^{-3} \mathrm{~g}$ RMS broadband and target vibration at $30 \mathrm{x}$

$1310^{-3} \mathrm{~g}$ RMS broadband; $\beta_{\mathrm{m}}=-\frac{\pi}{4} \mathrm{rad}$ ); mean a) amplitudes, b) dB reduction and c) phase difference]

14 [INSERT: Figure 8. Comparison between LDV measurement before and after correction with 'true' 15 target vibration (steering mirror vibration at $50 \times 10^{-3} \mathrm{~g}$ RMS broadband and target vibration at $60 \mathrm{x}$ $10^{-3} \mathrm{~g}$ RMS broadband; $\left.\beta_{\mathrm{m}}=-\frac{5 \pi}{12} \mathrm{rad}\right)$; mean a) amplitudes, b) dB reduction and c) phase difference]

\section{Experimental investigation - real world measurement scenario}

Taking the procedure out of the laboratory environment and into a real world application to tackle a particular challenge is the ultimate aim of such research. The specific industrial measurement described in this subsection was undertaken in response to a reported issue with significant handlebar vibration in a single cylinder racing motorcycle. In addition to making vibration measurements from the handlebars themselves and from various locations on the engine (using traditional piezoelectric accelerometers), the development team were interested to understand the mechanism by which the vibrational energy was transferred from the engine into the chassis and the front engine mounting was therefore of interest.

The motorcycle was to be tested in a dynamometer facility with restricted access to the area of interest as can be seen in Figure 9. Furthermore, the nature of the target surface (cylindrical with a circa 10 mm radius) made the attachment of an accelerometer at the preferred location and required orientation difficult, particularly in the space available. The logical choice of instrumentation was therefore a laser Doppler vibrometer, tripod-mounted on the isolated test cell floor with its beam steered through approximately $90^{\circ}$ by a suitably positioned mirror, to reach the required location with the required orientation. 
1 [INSERT: Figure 9. Experimental arrangement for real world racing motorcycle vibration

2 measurements (with laser beam path highlighted); a) overview and b) close-up]

3 Given the whole body motion of the motorcycle during (stepped) run-up/down, variable load testing,

4 maintaining the laser beam positioning was still non-trivial; the closer the mirror to the surface of

5 interest the better, of course. Given the significant vibration of the motorcycle transferred into the

6 dynamometer and any vibration generated within the dyno bed itself, the steering mirror was

7 experiencing significant vibration. This would normally lead to an erroneous measurement but,

8 according to the method presented in this paper, this can be corrected provided a measurement can be

9 simultaneously captured from the point of incidence of the laser beam on the mirror.

Inter-channel time delay adjustment for large frequency ranges and resulting measurement correction

11 In the interests of minimising the (per average) acquisition duration and since the ambition was to

12 gain an initial understanding of the nature of the system behaviour rather than to fully characterise it,

13 a frequency resolution of $10 \mathrm{~Hz}$ was selected. Given the requirement to investigate the system

14 dynamic behaviour over an extended rpm range and for orders up to $4 \mathrm{x}$ crank speed, the frequency

15 range of interest was defined as $5.12 \mathrm{kHz}$. This is much wider than the ranges used in earlier

16 laboratory experiments. For such a frequency range, the time delay between the mirror accelerometer

17 and LDV channels will result in the phase difference between the channels "wrapping" through $2 \pi$

18 radians as the frequency increases and this is a complication for the time delay calculation. Its

19 resolution is dealt with in Appendix B.

20 Proceeding with the post-processing of the measured signals, Figure 10 shows an example spectrum acquired during a top gear run-up at $5 \%$ load. It can be seen that, in this case, the mirror vibration is in the same order of magnitude to that of the target surface of interest up to approximately $150 \mathrm{~Hz}$, beyond which it drops off to insignificant levels. Using equation (13), the required correction to the LDV measurement is made with particular effect up to $150 \mathrm{~Hz}$, which includes the half engine order frequency that is important in a four stroke internal combustion engine.

[INSERT: Figure 10. Comparison between LDV measurement before and after correction for a spectrum obtained at 11605 rpm during a top gear, wide-open-throttle run-up at 5\% load; steering mirror vibration also shown for reference]

By post-processing further spectra obtained during the run-up, a waterfall order plot of the corrected LDV measurements made between 9703 and 11605 rpm at nominally 200 rpm intervals can be prepared, as shown in Figure 11. Such a data presentation format is common in powertrain NVH to show the evolution of engine order driven responses. In this particular plot, a structural resonance with a frequency close to $190 \mathrm{~Hz}$ can be observed, reaching a maximum when the first engine order coincides with it at circa $11605 \mathrm{rpm}$. The individual spectrum selected for Figure 10 is for the engine speed where the first engine order and the structural resonance coincide. These data revealed the 
1 reasons behind the reported excessive handlebar vibration. In this case, the team was able to make an

2 informed decision as to how best to overcome the problem by targeting the source, i.e. the engine,

3 and/or the transfer path, i.e. the chassis including the engine mounts through the frame to the

4 handlebars themselves.

5 [INSERT: Figure 11. Waterfall order plot showing corrected LDV measurements during a top-gear,

6 wide-open-throttle run-up at 5\% load from $9703-11605 \mathrm{rpm}$ ]

\section{$7 \quad$ 5. Further work: extension to accommodate steering mirror angular vibrations}

8 In the presence of angular mirror vibrations, the measurement of $\left(\hat{z}_{m} \cdot \overrightarrow{V_{B^{\prime}}}\right)$ is still exactly what is

9 required but there will be a small error between a calculation of $\left(\hat{b}_{1} \cdot \hat{n}_{B^{*}}\right)$ based on only the static

10 geometry and the actual (time-varying) quantity because the angular vibration causes a mirror

11 orientation dependent variation in $\hat{n}_{B *}$. From equation (6b) and with small angle approximations

12 applied (as set out in Appendix C):

$$
\left(\hat{b}_{1} \cdot \hat{n}_{B *}\right)=\left(\sin \beta_{m}+\Delta \theta_{m y} \cos \beta_{m}\right)
$$

14 in which the time-dependent error is evident in the term $\Delta \theta_{m y} \cos \beta_{m}$. This can be written in the form of an error, $e$ :

$$
e=\frac{\Delta \theta_{m y}}{\tan \beta_{m}}
$$

Note that this use of small angle approximations plays no part in the correction methods proposed; it is used primarily to provide the reader with clear insight into the principal source of the error $\left(\Delta \theta_{m y}\right)$, with quantification as shown in Figure 12. An RMS error per degree peak of angular vibration between $0.5 \%$ and $2 \%$ is indicated and, since the frequency content of the varying parts of $\hat{n}_{B *}$ and $\overrightarrow{V_{B^{\prime}}}$ is likely to be the same, this error would appear at the sum and difference frequencies i.e. at DC and $2 x$ vibration frequency.

[INSERT: Figure 12. RMS Error in correction velocity as a function of mirror orientation when steering mirror angular vibration is present] The size of the error is clearly dependent upon the amplitude of angular motion and the ultimate effect on the quality of the measurements is dependent on the relative scale of mirror and target vibrations. If further cancellation is required, however, this can be achieved by placing three transducers on the back-surface of the mirror equally spaced around a circle centred on the point of incidence of the laser beam. These measurements can provide the required component of translational vibration and the two components of angular vibration. 


\section{Mathematical determination of the required correction}

Using a vector-based approach and with reference to Figure 13, the arbitrary velocity at the location of the first transducer, $\overrightarrow{V_{1}}$, can be written in terms of the arbitrary velocity at the incident point $B^{\prime}, \overrightarrow{V_{B^{\prime}}}$, and the arbitrary angular motion around the chosen point, $\overrightarrow{\Delta \theta}$ :

$$
U_{1}=\hat{z}_{m} \cdot \overrightarrow{V_{1}}=\hat{z}_{m} \cdot\left(\overrightarrow{V_{B^{\prime}}}+\overrightarrow{r_{1}} \times \overrightarrow{\Delta \theta}\right)=\left(\hat{z}_{m} \cdot \overrightarrow{V_{B^{\prime}}}\right)+\overrightarrow{r_{1}} \cdot\left(\overrightarrow{\Delta \theta} \times \hat{z}_{m}\right)
$$

where $\overrightarrow{r_{1}}$ is the position vector for the accelerometer location relative to the chosen point along the laser beam path. A minimum of three similar independent equations is required to resolve the three components: $\left(\hat{z}_{m} \cdot \overrightarrow{V_{B^{\prime}}}\right), \Delta \theta_{m x}$ and $\Delta \theta_{m y}$.

$$
\begin{aligned}
& U_{2}=\hat{z}_{m} \cdot \overrightarrow{V_{2}}=\hat{z}_{m} \cdot\left(\overrightarrow{V_{B^{\prime}}}+\overrightarrow{r_{2}} \times \overrightarrow{\Delta \theta}\right)=\left(\hat{z}_{m} \cdot \overrightarrow{V_{B^{\prime}}}\right)+\overrightarrow{r_{2}} \cdot\left(\overrightarrow{\Delta \theta} \times \hat{z}_{m}\right) \\
& U_{3}=\hat{z}_{m} \cdot \overrightarrow{V_{3}}=\hat{z}_{m} \cdot\left(\overrightarrow{V_{B^{\prime}}}+\overrightarrow{r_{3}} \times \overrightarrow{\Delta \theta}\right)=\left(\hat{z}_{m} \cdot \overrightarrow{V_{B^{\prime}}}\right)+\overrightarrow{r_{3}} \cdot\left(\overrightarrow{\Delta \theta} \times \hat{z}_{m}\right)
\end{aligned}
$$

The quantity $\left(\hat{z}_{m} \cdot \overrightarrow{V_{B^{\prime}}}\right)$ is obtained by summing the three measurements with locations such that:

$$
\overrightarrow{r_{1}}+\overrightarrow{r_{2}}+\overrightarrow{r_{3}}=0
$$

and this can be achieved with the three transducers equally spaced around a circle, for example as shown in Figure 13, in which:

$$
\overrightarrow{r_{1}}=r \hat{y}_{m}
$$

such that equations (16a-c) can be reorganised as follows:

$$
\begin{aligned}
& U_{1}=\left(\hat{z}_{m} \cdot \overrightarrow{V_{B^{\prime}}}\right)+\overrightarrow{\Delta \theta} \cdot\left(\hat{z}_{m} \times \overrightarrow{r_{1}}\right)=\left(\hat{z}_{m} \cdot \overrightarrow{V_{B^{\prime}}}\right)-r\left(\overrightarrow{\Delta \theta} \cdot \hat{x}_{m}\right) \\
& U_{2}=\left(\hat{z}_{m} \cdot \overrightarrow{V_{B^{\prime}}}\right)+\overrightarrow{\Delta \theta} \cdot\left(\hat{z}_{m} \times \overrightarrow{r_{2}}\right)=\left(\hat{z}_{m} \cdot \overrightarrow{V_{B^{\prime}}}\right)+r\left(\frac{1}{2} \overrightarrow{\Delta \theta} \cdot \hat{x}_{m}+\frac{\sqrt{3}}{2} \overrightarrow{\Delta \theta} \cdot \hat{y}_{m}\right) \\
& U_{3}=\left(\hat{z}_{m} \cdot \overrightarrow{V_{B^{\prime}}}\right)+\overrightarrow{\Delta \theta} \cdot\left(\hat{z}_{m} \times \overrightarrow{r_{3}}\right)=\left(\hat{z}_{m} \cdot \overrightarrow{V_{B^{\prime}}}\right)+r\left(\frac{1}{2} \overrightarrow{\Delta \theta} \cdot \hat{x}_{m}-\frac{\sqrt{3}}{2} \overrightarrow{\Delta \theta} \cdot \hat{y}_{m}\right)
\end{aligned}
$$

[INSERT: Figure 13. Suitable arrangement of three accelerometers on the back face of a steering mirror to make measurements for correction of translational and angular mirror vibration]

From these equations the required components can be resolved without approximations:

$$
\begin{aligned}
& \left(\hat{z}_{m} \cdot \overrightarrow{V_{B^{\prime}}}\right)=\frac{1}{3}\left(U_{1}+U_{2}+U_{3}\right) \\
& \Delta \theta_{m x}=\left(\overrightarrow{\Delta \theta} \cdot \hat{x}_{m}\right)=\frac{1}{3 r}\left(-2 U_{1}+U_{2}+U_{3}\right)
\end{aligned}
$$




$$
\Delta \theta_{m y}=\left(\overrightarrow{\Delta \theta} \cdot \hat{y}_{m}\right)=\frac{\sqrt{3}}{3 r}\left(U_{2}-U_{3}\right)
$$

2 Substituting equations (19b\&c) into equation (6b) allows the time-dependent $\hat{n}_{B *}$ to be written in the global coordinate system based on the measurements made. This in turn allows the time-dependent scalar product $\left(\hat{b}_{1}, \hat{n}_{B *}\right)$ in equation (14) to be calculated for an improved correction of the steering mirror vibration. Experimental implementation of this proposed scheme will be the subject of further work.

\section{Conclusions}

When an optical device, typically a planar mirror, is used to steer the laser beam of an LDV to an inaccessible location but is itself subject to vibration, the measurements made will be sensitive to any vibration of the mirror in the direction of its normal. This paper has confirmed this sensitivity, for the first time, through a rigorous theoretical and experimental study, and has demonstrated that correction can be conveniently made using appropriately located and oriented mirror vibration measurements scaled according to mirror orientation. While measurements including steering optics are relatively commonplace, in particular for measurements on surfaces where access is restricted, e.g. within complex machines such as reciprocating engines, this is the first time that the extent of the impact of steering optic vibration and correction for it have been formalised and reported.

Experimental validation of the measurement correction in a laboratory environment showed error reductions of between $33 \mathrm{~dB}$ and $47 \mathrm{~dB}$ for measurements with various mirror:target vibration level ratios and mirror orientation angles. Corresponding RMS phase errors were around $30 \mathrm{mrad}$. A previously unreported real-world measurement on a single cylinder racing motorcycle engine mount, in which a steering mirror was mounted to a vibrating test cell platform, was used to demonstrate the applicability and practicality of the technique. Insights into the nature of the system vibratory behaviour, which would otherwise have been obscured by the significant vibration of the mirror, were identified and described.

The work described in this paper is of fundamental importance to the vibration measurement community. For the first time, accuracy has been restored to LDV measurements enabled by beam steering optics that are themselves unavoidably subject to vibration. Rather than having either to compromise on the measurement point of interest or to rely on an imperfectly vibration isolated steering optic, it is now possible to make fully corrected measurements using a validated scheme that is straightforward to implement in practice. Successful application of the correction method, as part of a study of a motorcycle engine with a high reported vibration level, demonstrates its suitability for challenging real-world applications. 


\section{Appendices}

\section{A: Rotation matrices}

$3 \quad[x, \alpha]=\left[\begin{array}{ccc}1 & 0 & 0 \\ 0 & \cos \alpha & -\sin \alpha \\ 0 & \sin \alpha & \cos \alpha\end{array}\right]$

$4 \quad[y, \beta]=\left[\begin{array}{ccc}\cos \beta & 0 & \sin \beta \\ 0 & 1 & 0 \\ -\sin \beta & 0 & \cos \beta\end{array}\right]$

\section{B: Estimating inter-channel time delay across an extended frequency range}

6 Data were collected during the standard pre-test experiment in which the steering mirror

7 accelerometer was mounted on a shaker undergoing broadband vibration across the frequency range

8 of interest with the vibrometer making a simultaneous measurement from the top surface of the

9 accelerometer in its sensitive direction. This experiment serves to check amplitude sensitivity and to

10 calculate the inter-channel time delay. This appendix is focussed on the estimation of inter-channel

11 time delay across a frequency range broad enough that phase-wrapping occurs as shown in Figure B 1

12 (frequency range capped at $3 \mathrm{kHz}$ for brevity). It is possible to use only the frequency range up to the

13 first wrap, as is the case for the data in Figure 3, to estimate inter-channel time delay but it is better to

14 use the whole frequency range if possible.

15 [INSERT: Figure B 1. Example plot of phase difference between steering mirror accelerometer signal 16 (integrated for velocity) and LDV signal, showing progression from initial phase-wrapped calculation 17 to fully unwrapped calculation]

18 To unwrap the calculation of phase difference (dotted curve in Figure B 1), an algorithm was

19 developed to identify each wrap event. This compared the current phase difference value with the

20 previous value, with a wrap identified where that comparison exceeded a set threshold. A further $2 \pi$

21 radians was then subtracted from the difference. The performance of the algorithm is affected by the

22 level of noise in the signals and this required some manual intervention. The aim was to select a

23 suitable threshold value which could be used for all averages but occasionally phase difference spikes

24 due to noise resulted in a phase wrap being incorrectly detected (dot-dash curve in Figure B 1).

25 Examples of when this occurred are shown at 830 and $1770 \mathrm{~Hz}$ in Figure B 1. Manual intervention

26 was required with the final result being an unwrapped phase difference, as shown in the dashed line of

27 Figure B 1 , whose gradient is given by $2 \pi \tau$, where $\tau$ is the required time delay. With the time delay

28 then accommodated ( $\tau=1.3 \mathrm{~ms}$ ), the phase difference between the true and corrected measurements

29 is shown as the solid line. 
$\hat{n}_{B *}$

$=\left[\begin{array}{ccc}\hat{x} & \hat{y} & \hat{z}\end{array}\right]\left[\begin{array}{ccc}1 & 0 & 0 \\ 0 & \cos \alpha_{m}+\Delta \theta_{m x} & -\sin \alpha_{m}+\Delta \theta_{m x} \\ 0 & \sin \alpha_{m}+\Delta \theta_{m x} & \cos \alpha_{m}+\Delta \theta_{m x}\end{array}\right]\left[\begin{array}{ccc}\cos \beta_{m}+\Delta \theta_{m y} & 0 & \sin \beta_{m}+\Delta \theta_{m y} \\ 0 & 1 & 0 \\ -\sin \beta_{m}+\Delta \theta_{m y} & 0 & \cos \beta_{m}+\Delta \theta_{m y}\end{array}\right]\left[\begin{array}{l}0 \\ 0 \\ 1\end{array}\right]$

$3 \quad \hat{n}_{B *}=\left[\begin{array}{lll}\hat{x} & \hat{y} & \hat{z}\end{array}\right]\left[\begin{array}{c}\sin \left(\beta_{m}+\Delta \theta_{m y}\right) \\ -\sin \left(\alpha_{m}+\Delta \theta_{m x}\right) \cos \left(\beta_{m}+\Delta \theta_{m y}\right) \\ \cos \left(\beta_{m}+\Delta \theta_{m y}\right) \cos \left(\beta_{m}+\Delta \theta_{m y}\right)\end{array}\right]$

$4 \quad$ With small angle approximations:

$5 \quad\left(\hat{b}_{1} \cdot \hat{n}_{B *}\right)=\left(\sin \beta_{m}+\Delta \theta_{m y} \cos \beta_{m}\right)$

6 Acknowledgements

7 The authors wish to acknowledge the support of GE Precision Engineering (http://www.geprecision-

8 engineering.co.uk) for reporting and allowing access to the racing motorcycle vibration investigation.

\section{REFERENCES}

[1] S.J. Rothberg, M.S. Allen, P. Castellini, D. Di Maio, J.J.J. Dirckx, D.J. Ewins, B.J. Halkon, P. Muyshondt, N. Paone, T. Ryan, H. Steger, E.P. Tomasini, S. Vanlanduit, and J.F. Vignola, An international review of laser Doppler vibrometry: making light work of vibration measurement, Optics and Lasers in Engineering (2016). DOI: 10.1016/j.optlaseng.2016.10.023

[2] A. Carlini, A. Rivola, G. Dalpiaz and A. Maggiore, Valve motion measurements on motorbike cylinder heads using high-speed laser vibrometer. Proc. 5th Int. Conf. on Vib. Meas. by Laser Techs. (2002) 564-574.

[3] J.G. Sczepanski, New equipment and methodology to perform high speed valvetrain dynamics testing and analysis (2004-01-1720). SAE Technical Paper (2004).

[4] R. Kerres, D. Schwarz, M. Bach, K. Fuoss, A. Eichenberg and J. Wüst, Overview of measurement technology for valve lift and rotation on motored and fired engines (2012-010159). SAE Int. J. Engines, (2012) 197-206.

[5] B.J. Halkon and S.J. Rothberg, Correction of laser Doppler vibrometry measurements affected by steering mirror vibration, Proc. OPTIMESS III (2016) 117-126.

[6] B.J. Halkon and S.J. Rothberg, Taking laser Doppler vibrometry off the tripod: correction of measurements affected by instrument vibration, Optics and Lasers in Engineering 91 (2017) 16-23. DOI: 10.1016/j.optlaseng.2016.11.006

[7] S.J. Rothberg and M. Tirabassi, A universal framework for modelling measured velocity in laser vibrometry with applications. Mech. Syst. Sig. Proc. 26 (2012) 141-166. 


\section{Figure captions}

Figure 1. Schematic showing translational vibration measurement using an angled steering mirror; beam deviation significantly exaggerated for the sake of clarity

Figure 2. Equipment arrangement for laboratory-based experimental investigation; laser beam path highlighted for the sake of clarity

Figure 3. Example phase difference between LDV measurement and integrated accelerometer measurement before and after time delay adjustment

Figure 4. Comparison between measurements from the LDV and from the target accelerometer on a (nominally) stationary target (beam steered through $90^{\circ}$ by the mirror with $\beta_{\mathrm{m}}=-\frac{\pi}{4}$ rad; mirror vibration at $50 \times 10^{-3} \mathrm{~g}$ RMS broadband)

Figure 5. Comparison between measured and corrected LDV measurements with respect to the 'true' target vibration (stationary target, beam steered through $90^{\circ}$ by the mirror with $\beta_{\mathrm{m}}=-\frac{\pi}{4} \mathrm{rad}$; mirror vibration at $50 \times 10^{-3} \mathrm{~g}$ RMS broadband); mean a) amplitudes and b) $\mathrm{dB}$ reduction

Figure 6. Comparison between measured and corrected LDV measurements with respect to the 'true' target vibration (stationary target, mirror vibration at $50 \times 10^{-3} \mathrm{~g}$ RMS broadband); a) beam steered through $60^{\circ}$, i.e. $\beta_{\mathrm{m}}=-\frac{\pi}{3} \mathrm{rad}$, b) beam steered through $30^{\circ}$, i.e. $\beta_{\mathrm{m}}=-\frac{5 \pi}{12} \mathrm{rad}$ and c) dB reduction Figure 7. Comparison between LDV measurement before and after correction with 'true' target vibration (steering mirror vibration at $50 \times 10^{-3} \mathrm{~g}$ RMS broadband and target vibration at $30 \times 10^{-3} \mathrm{~g}$ RMS broadband; $\beta_{\mathrm{m}}=-\frac{\pi}{4} \mathrm{rad}$ ); mean a) amplitudes, b) dB reduction and c) phase difference Figure 8. Comparison between LDV measurement before and after correction with 'true' target vibration (steering mirror vibration at $50 \times 10^{-3} \mathrm{~g}$ RMS broadband and target vibration at $60 \times 10^{-3} \mathrm{~g}$ RMS broadband; $\beta_{\mathrm{m}}=-\frac{5 \pi}{12} \mathrm{rad}$ ); mean a) amplitudes, b) dB reduction and c) phase difference Figure 9. Experimental arrangement for real world racing motorcycle vibration measurements (with laser beam path highlighted); a) overview and b) close-up

Figure 10. Comparison between LDV measurement before and after correction for a spectrum obtained at 11605 rpm during a top gear, wide-open-throttle run-up at 5\% load; steering mirror vibration also shown for reference

Figure 11. Waterfall order plot showing corrected LDV measurements during a top-gear, wide-openthrottle run-up at 5\% load from 9703 - 11605 rpm

Figure 12. RMS Error in correction velocity as a function of mirror orientation when steering mirror angular vibration is present 
Figure 13. Suitable arrangement of three accelerometers on the back face of a steering mirror to make measurements for correction of translational and angular mirror vibration

Figure B 1. Example plot of phase difference between steering mirror accelerometer signal (integrated for velocity) and LDV signal, showing progression from initial phase-wrapped calculation to fully unwrapped calculation 
Figures

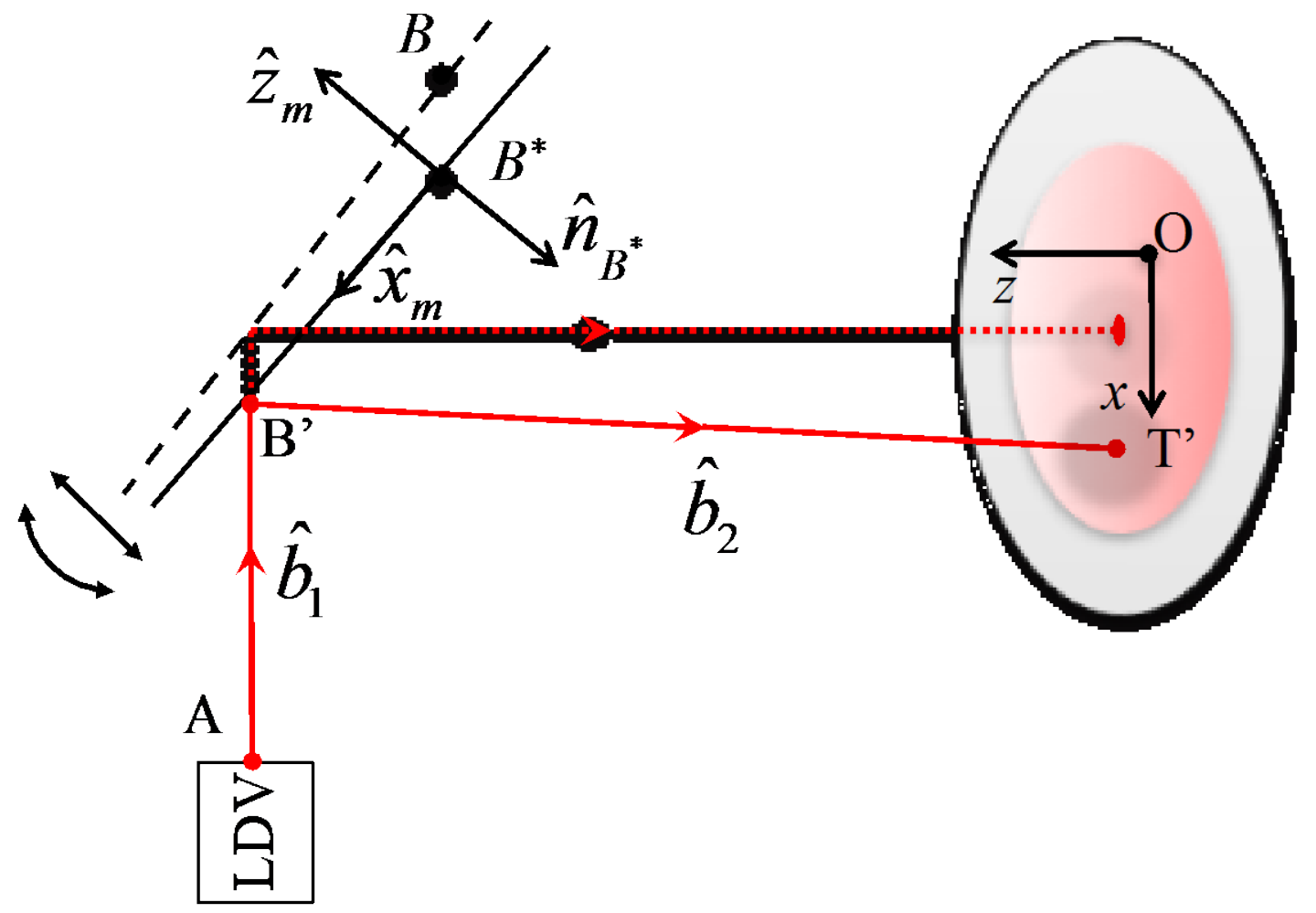

Figure 1. Schematic showing translational vibration measurement using an angled steering mirror; beam deviation significantly exaggerated for the sake of clarity 


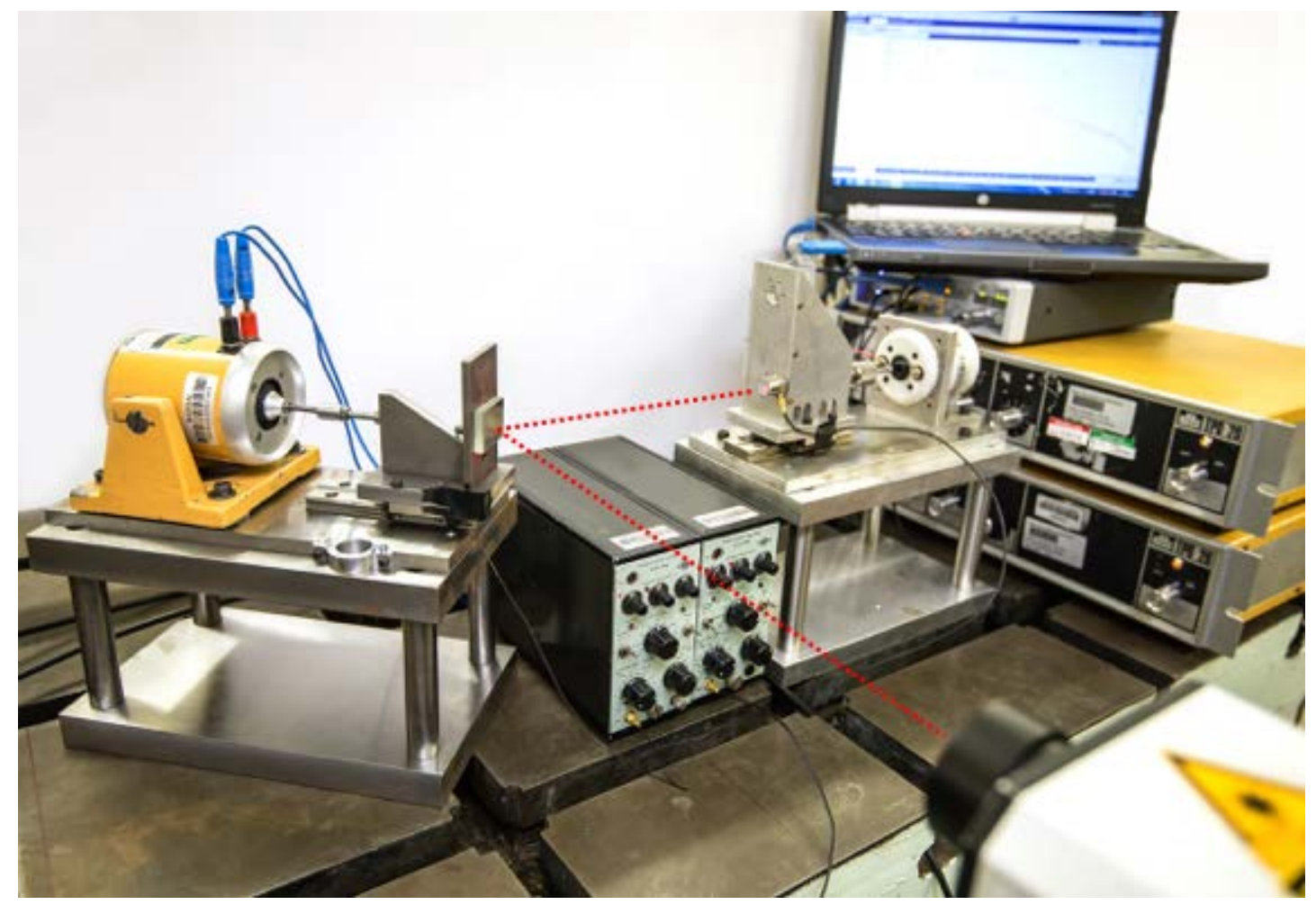

Figure 2. Equipment arrangement for laboratory-based experimental investigation; laser beam path highlighted for the sake of clarity

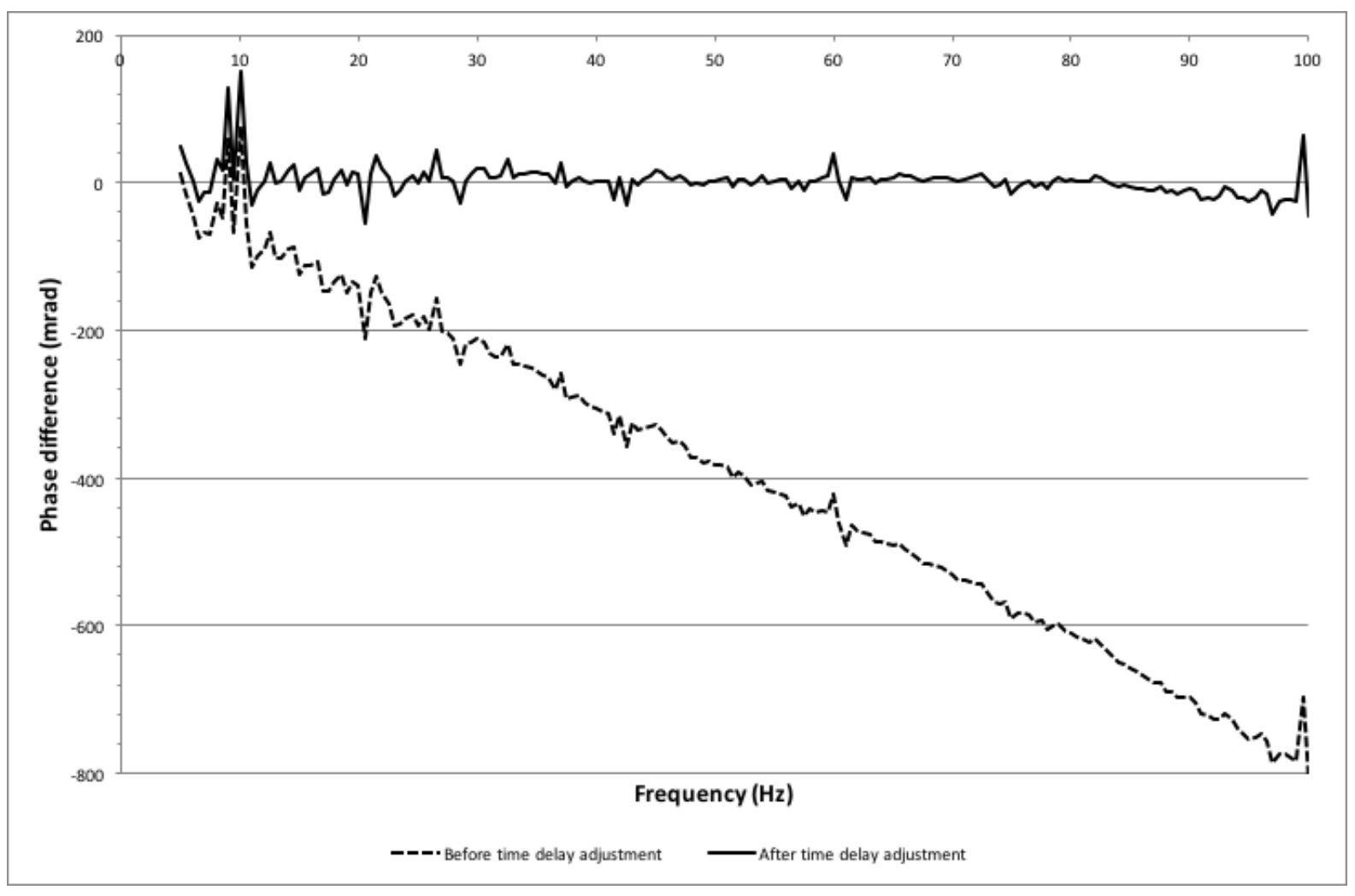

Figure 3. Example phase difference between LDV measurement and integrated accelerometer measurement before and after time delay adjustment 


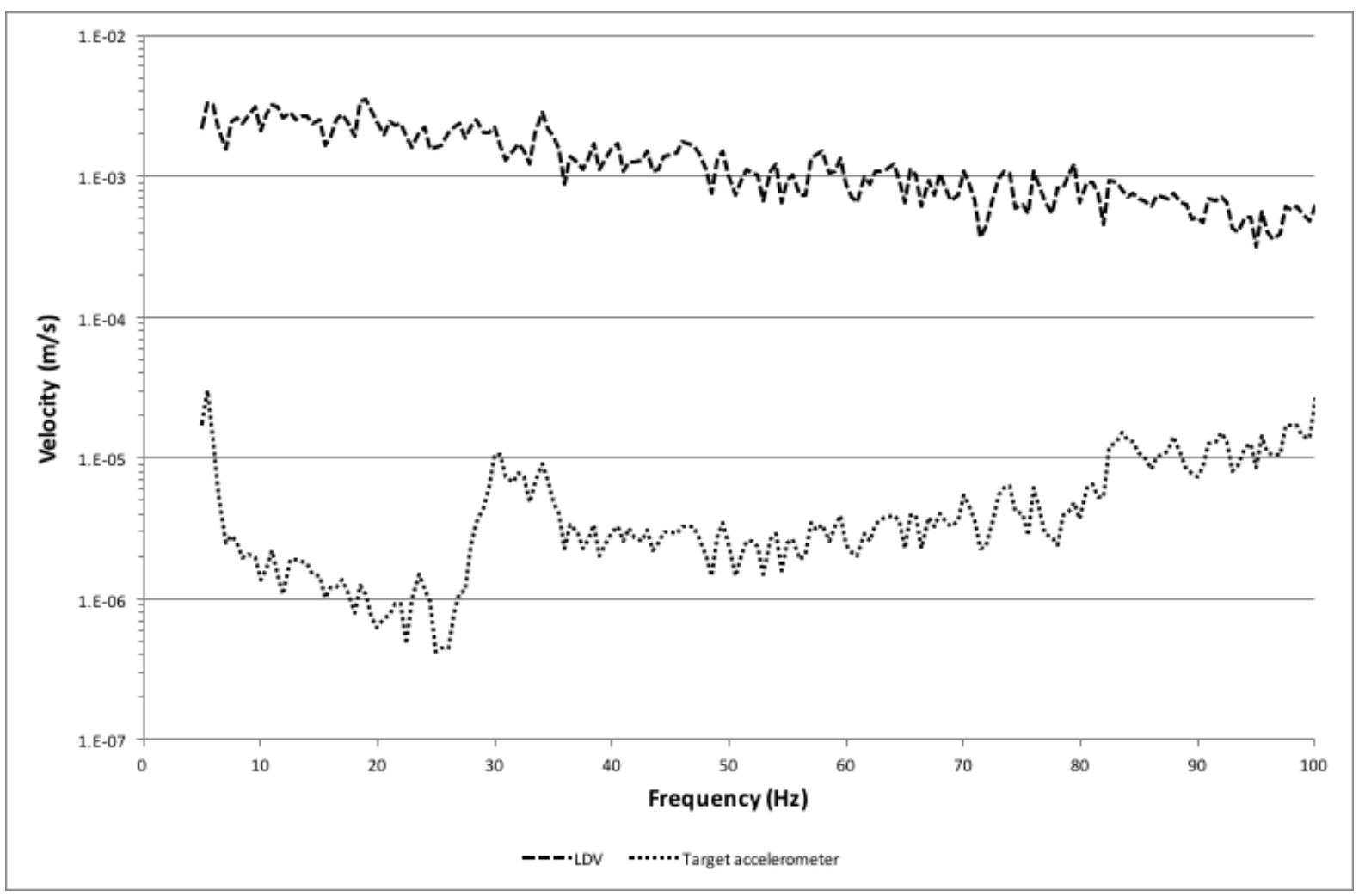

Figure 4. Comparison between measurements from the LDV and from the target accelerometer on a (nominally) stationary target (beam steered through $90^{\circ}$ by the mirror with $\beta_{m}=-\frac{\pi}{4}$ rad; mirror vibration at $50 \times 10^{-3} \mathrm{~g}$ RMS broadband)
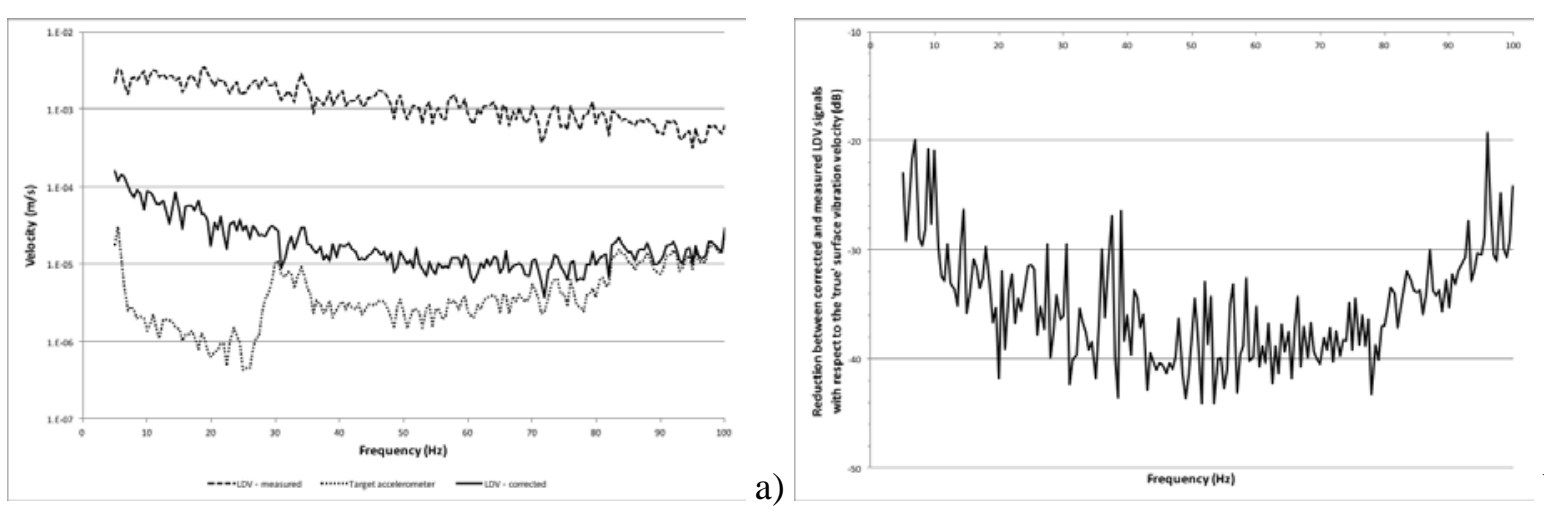

b)

Figure 5. Comparison between measured and corrected LDV measurements with respect to the 'true' target vibration (stationary target, beam steered through $90^{\circ}$ by the mirror with $\beta_{m}=-\frac{\pi}{4}$ rad; mirror vibration at $50 \times 10^{-3} \mathrm{~g}$ RMS broadband); mean a) amplitudes and b) dB reduction 

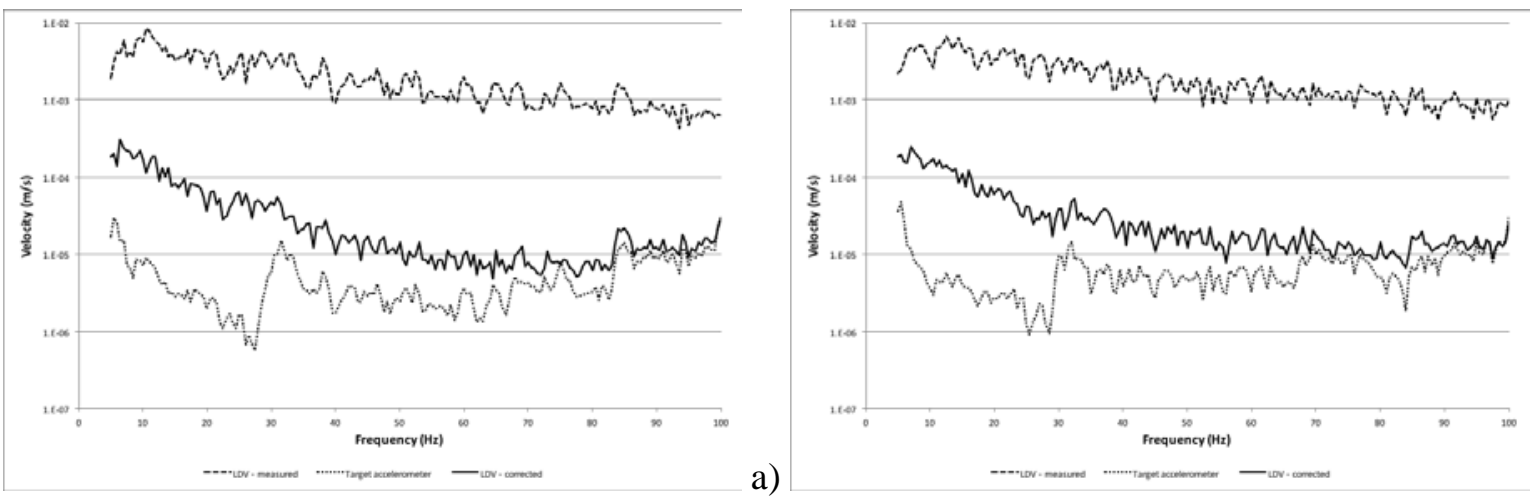

a)

b)

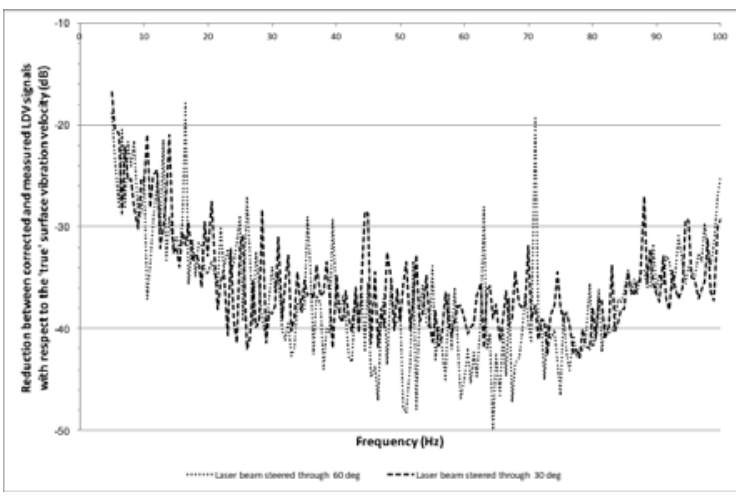

c)

Figure 6. Comparison between measured and corrected LDV measurements with respect to the 'true' target vibration (stationary target, mirror vibration at $50 \times 10^{-3} \mathrm{~g}$ RMS broadband); a) beam steered through $60^{\circ}$, i.e. $\beta_{m}=-\frac{\pi}{3} \mathrm{rad}$, b) beam steered through $30^{\circ}$, i.e. $\beta_{m}=-\frac{5 \pi}{12} \mathrm{rad}$ and c) $\mathrm{dB}$ reduction 

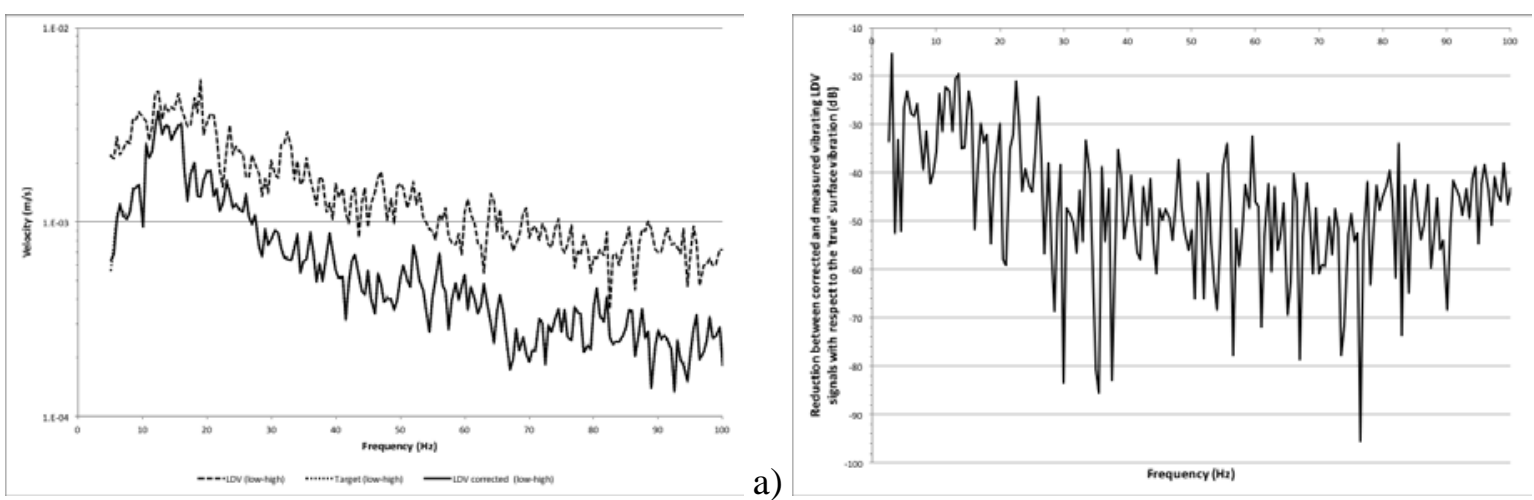

a)

b)

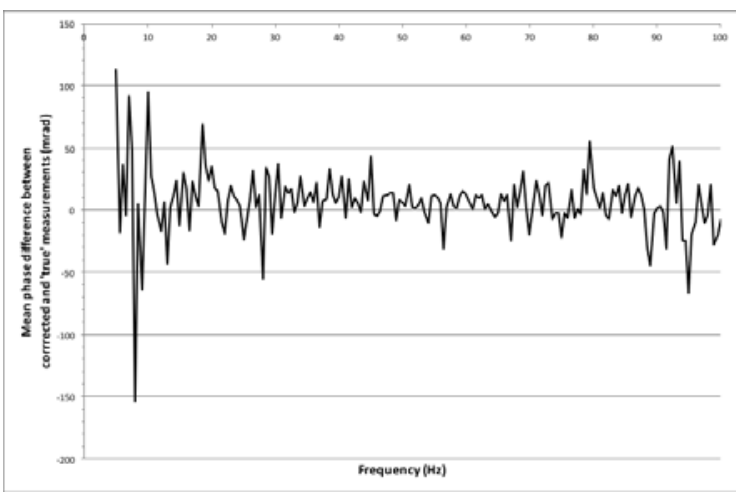

C)

Figure 7. Comparison between LDV measurement before and after correction with 'true' target vibration (steering mirror vibration at $50 \times 10^{-3} \mathrm{~g}$ RMS broadband and target vibration at $30 \times 10^{-3} \mathrm{~g}$ RMS broadband; $\beta_{m}=-\frac{\pi}{4}$ rad); mean a) amplitudes, b) dB reduction and c) phase difference 

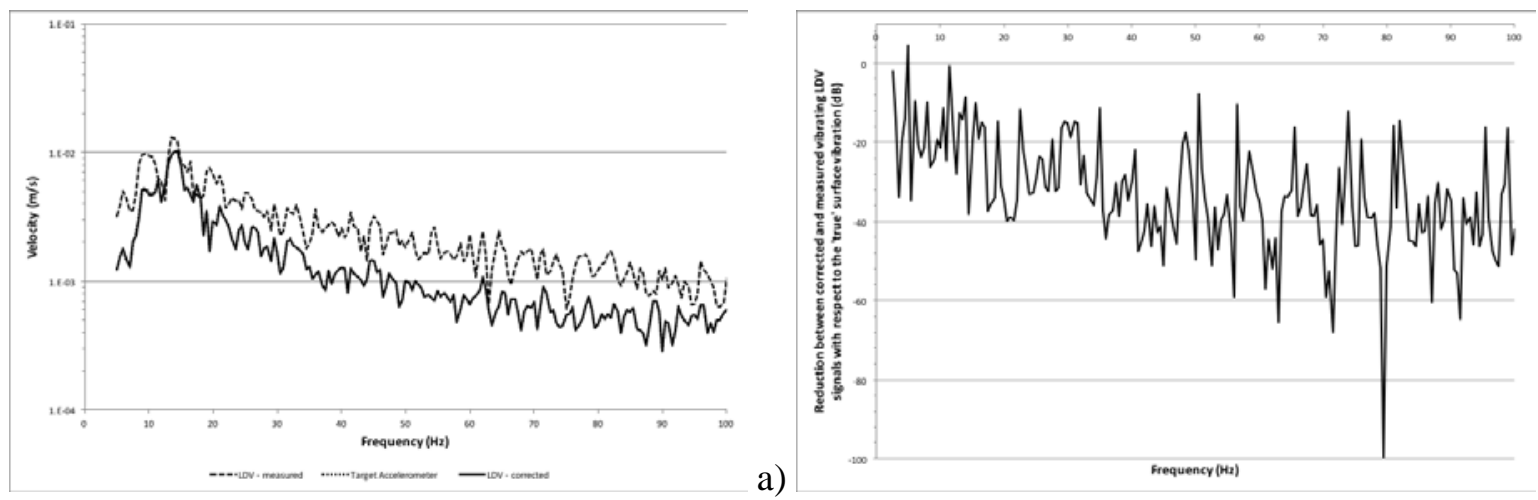

a)

b)

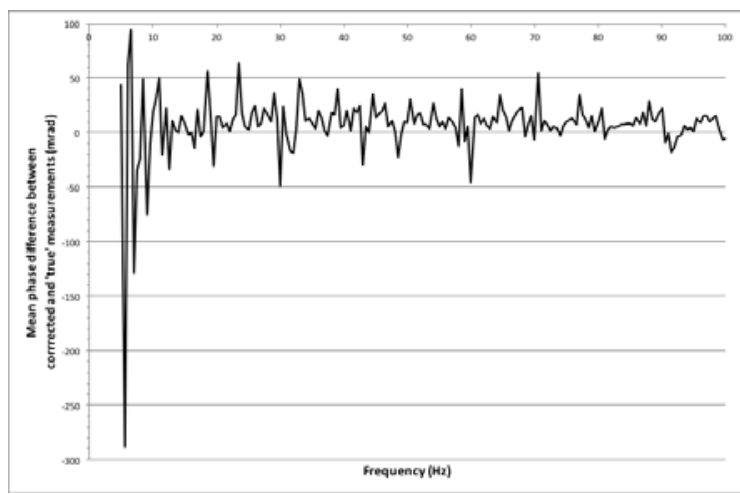

C)

Figure 8. Comparison between LDV measurement before and after correction with 'true' target vibration (steering mirror vibration at $50 \times 10^{-3} \mathrm{~g}$ RMS broadband and target vibration at $60 \times 10^{-3} \mathrm{~g}$ RMS broadband; $\beta_{m}=-\frac{5 \pi}{12} \mathrm{rad);} \mathrm{mean} \mathrm{a)} \mathrm{amplitudes,} \mathrm{b)} \mathrm{dB} \mathrm{reduction} \mathrm{and} \mathrm{c)} \mathrm{phase} \mathrm{difference}$
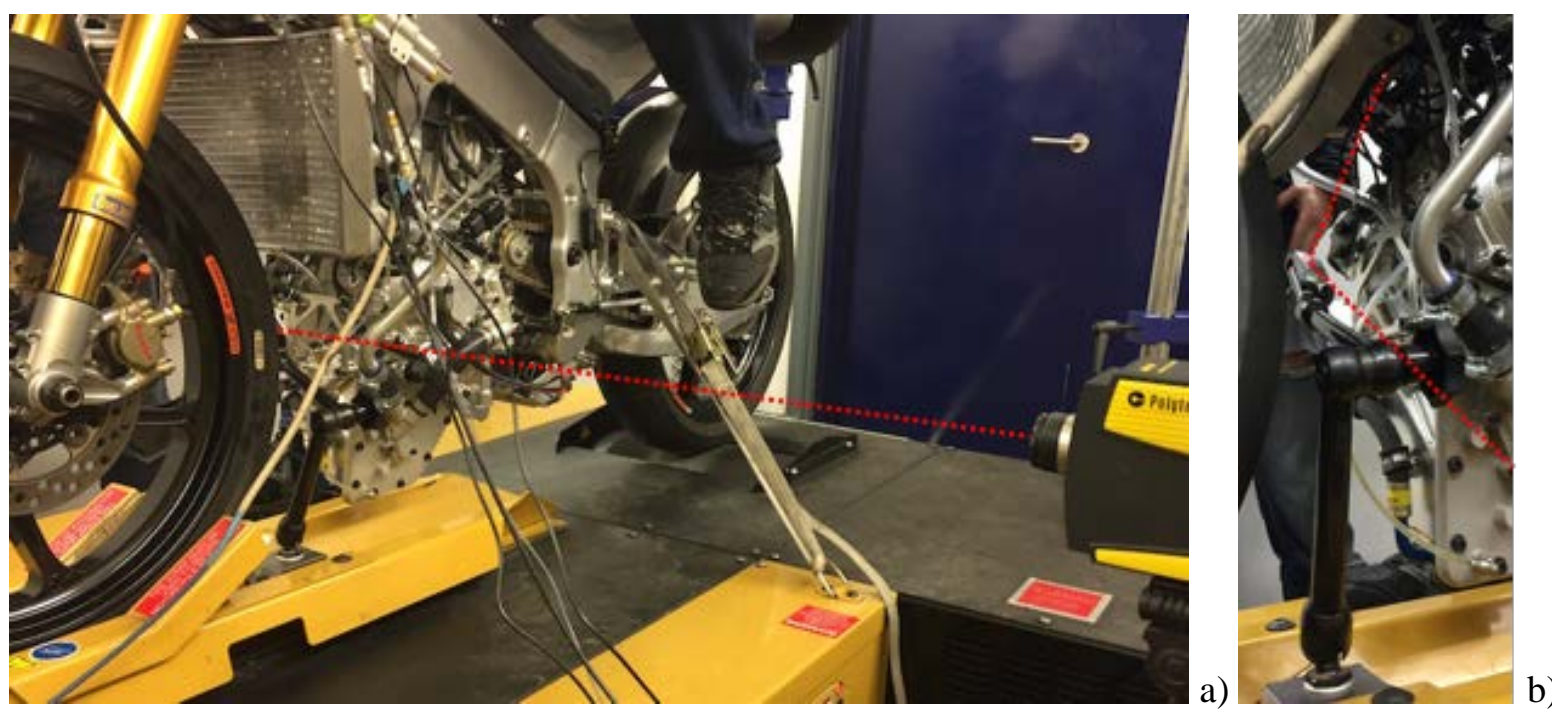

Figure 9. Experimental arrangement for real world racing motorcycle vibration measurements (with laser beam path highlighted); a) overview and b) close-up 


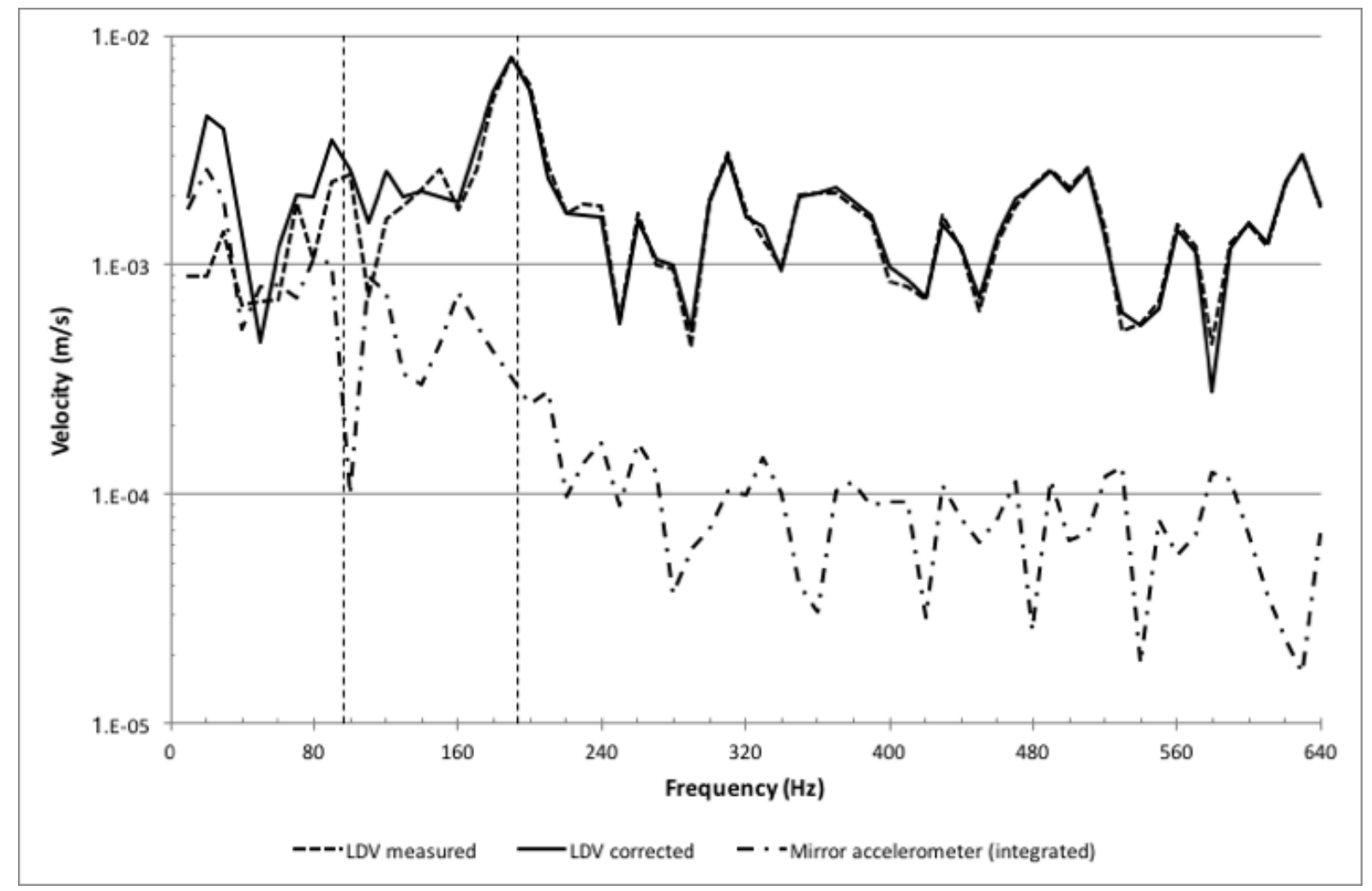

Figure 10. Comparison between LDV measurement before and after correction for a spectrum obtained at 11605 rpm during a top gear, wide-open-throttle run-up at 5\% load; steering mirror vibration also shown for reference

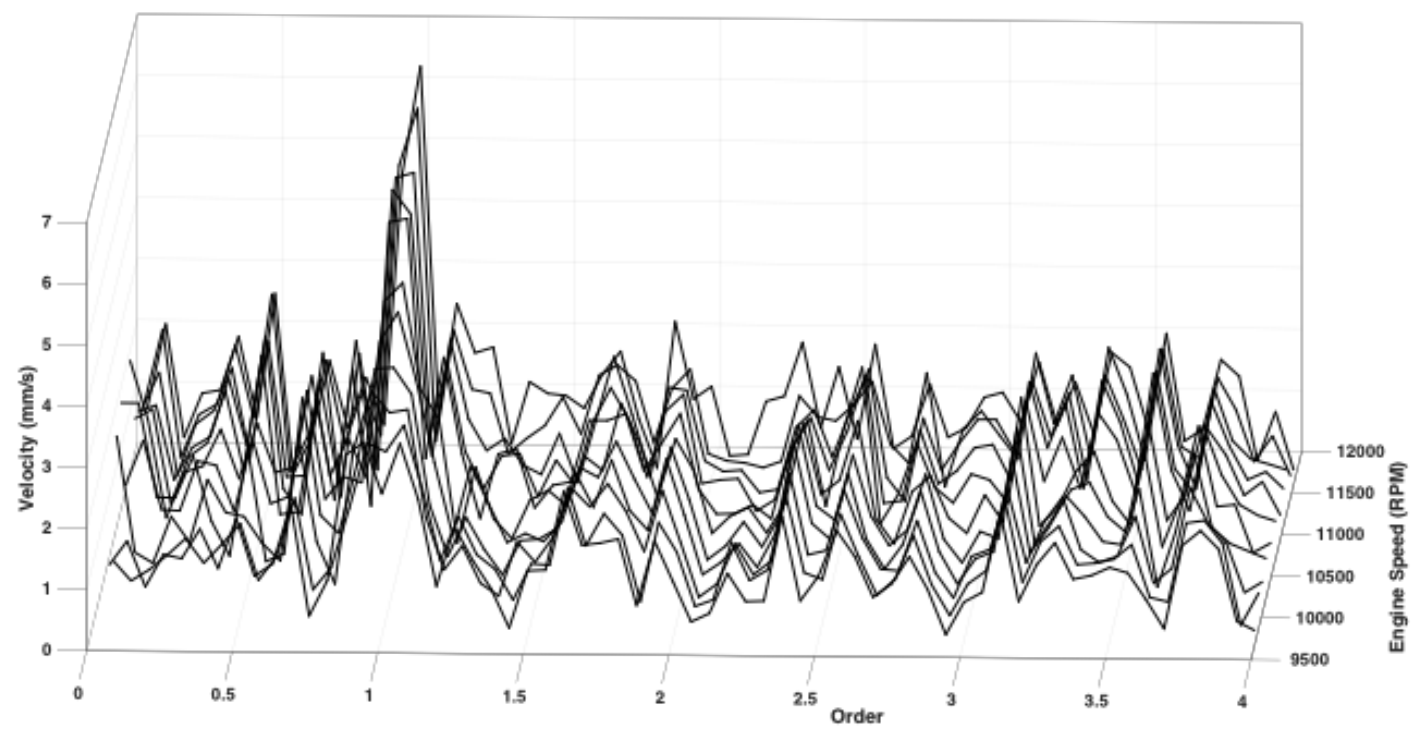

Figure 11. Waterfall order plot showing corrected LDV measurements during a top-gear, wide-openthrottle run-up at 5\% load from 9703 - 11605 rpm 


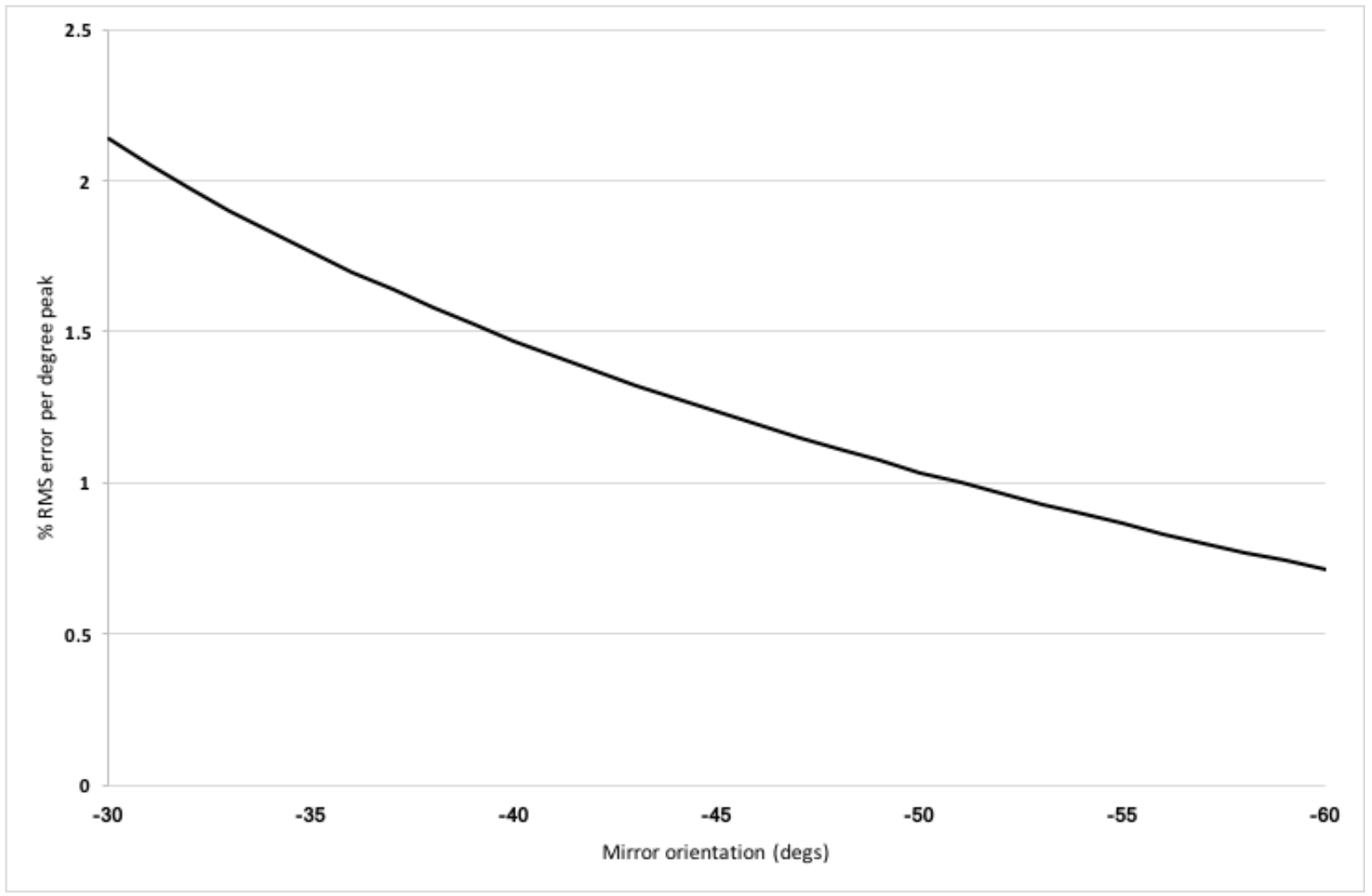

Figure 12. RMS Error in correction velocity as a function of mirror orientation when steering mirror angular vibration is present 


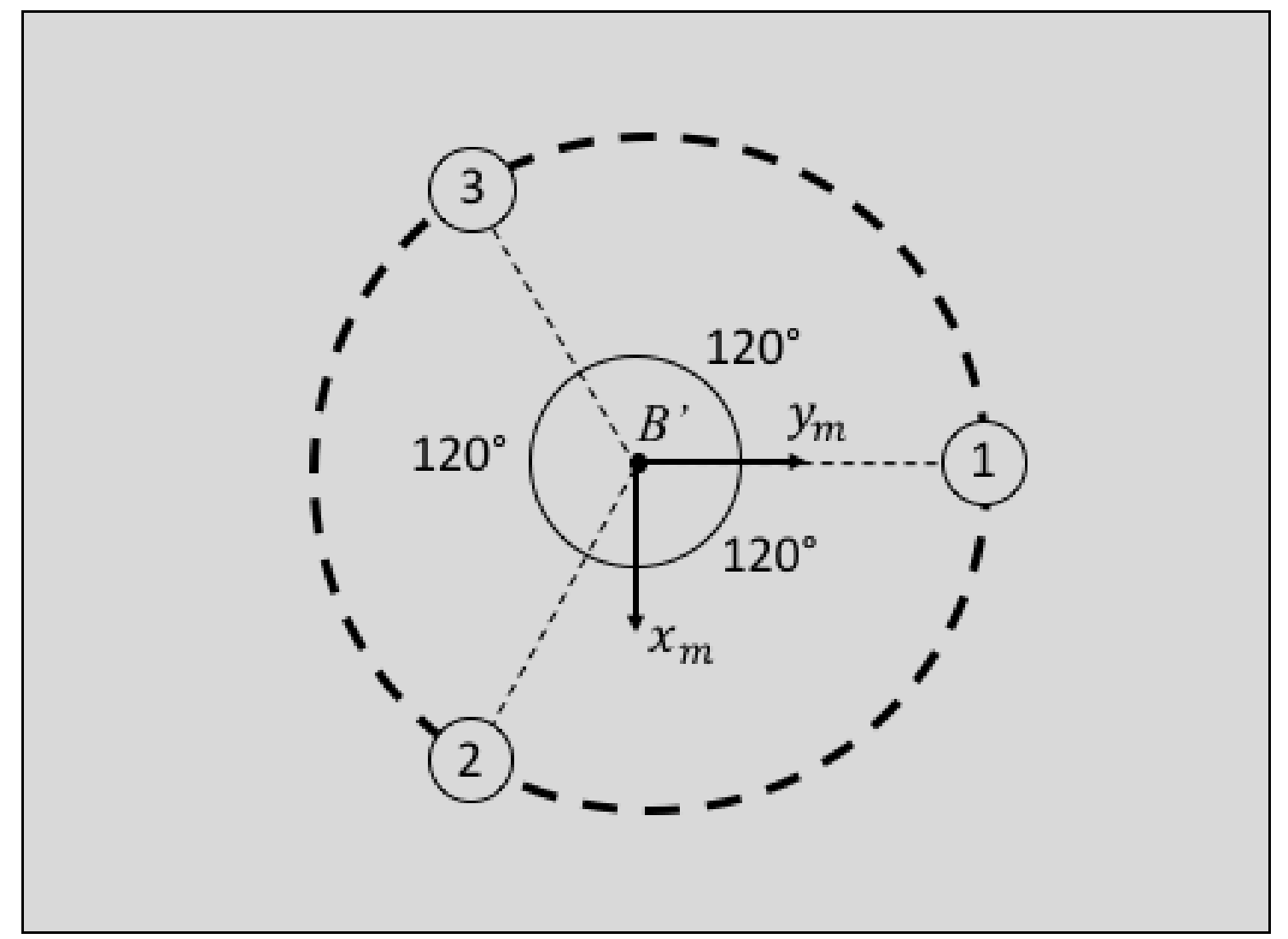

Figure 13. Suitable arrangement of three accelerometers on the back face of a steering mirror to make measurements for correction of translational and angular mirror vibration 


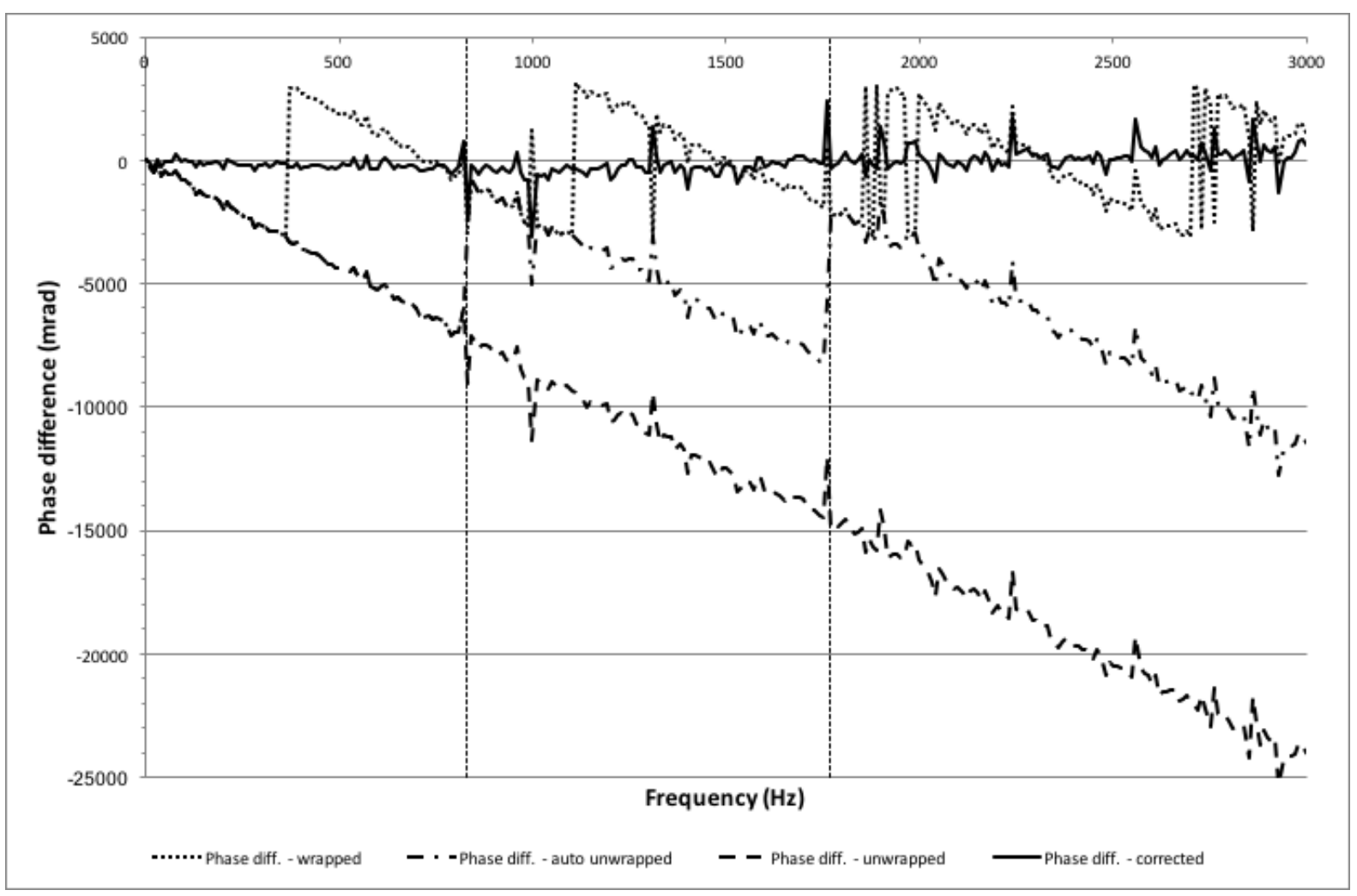

Figure B 1. Example plot of phase difference between steering mirror accelerometer signal (integrated for velocity) and LDV signal, showing progression from initial phase-wrapped calculation to fully unwrapped calculation 